\title{
Corner singularities in bi-material Mindlin plates
}

\author{
C.S. Huang * \\ Department of Civil Engineering, National Chiao Tung University, 1001 Ta-Hsueh Rd., Hsinchu 30050, Taiwan, ROC
}

\begin{abstract}
An eigenfunction expansion solution is first developed to find stress singualrities for bi-material wedges by directly solving the governing equations of the Mindlin plate theory in terms of displacement components. The singularity orders of moments and shear forces at corners are determined from the corresponding asymptotic solutions having the lowest order in $r$ and satisfying the radial boundary conditions and continuity conditions. The present solution is applied to thoroughly examine the singularities occurring at the interface joint of bonded dissimilar isotropic plates and at the vertex of a bi-material wedge with two simply supported radial edges. The corresponding characteristic equations for determining the singularity orders of moments and shear forces are explicitly given. The singularity orders of moment are shown in graphic form as functions of the flexural rigidity ratio and corner angle, while the shear force singularity orders are given as functions of the corner angle and the shear modulus ratio multiplied by the thickness ratio. The order of moment singularity obtained here for bonded dissimilar plates is also compared with that based on the classical plate theory. (C) 2002 Elsevier Science Ltd. All rights reserved.
\end{abstract}

Keywords: Stress singularities; Eigenfunction expansion; Bi-material plates; Mindlin plate theory

\section{Introduction}

It is well known that stress singularities arise in the mathematical solutions of plate problems, which can be due to concentrated forces and moments, discontinuities in edge conditions, or sharp corners. It has been pointed out and numerically shown that if singularities due to discontinuities in edge conditions or sharp corners are not properly considered in numerical solutions, significant errors will occur in the calculated global behavior of plates, such as static deflection, free vibration frequencies, forced dynamic response, and critical buckling load [1-4].

Williams $[5,6]$ was the first to investigate corner stress singularities in isotropic plates under bending or extension, due to various homogeneous boundary conditions. The investigation of corner stress singularities has also been extended to non-isotropic thin plates $[7,8]$, composite plates [9], isotropic thick plates [10,11], and isotropic three-dimensional problems [12]. The stress singularity at a crack tip is a special case examined in the above studies.

Some studies have also been carried out to investigate stress singularities in a bi-material wedge. Applying

\footnotetext{
${ }^{*}$ Tel.: +886-3-571-2121x54962.

E-mail address: cshuang@cc.nctu.edu.tw (C.S. Huang).
}

Mellin transformation to solve plane elasticity equations, Hein and Erdogan [13] investigated stress singularities at a bi-material corner, while Bogy and Wang [14] examined stress singularities at an interface corner in bonded dissimilar isotropic materials. Rao [15] used a solution technique similar to William's [5] to study singularities in the solution of a bi-harmonic differential equation due to interface conditions and boundary conditions. Dempsey and Sinclair [16] applied a new form of the Airy stress function to plane elasticity equations and investigated the singular behavior at the vertex of a bi-material wedge. Xie and Chaudhuri [17] proposed an eigenfunction expansion method for analyzing a three-dimensional problem of a bi-material wedge. Notably, the methodology used to solve these plane elasticity problems and some of their results can be directly applied to bending problems of thin plates because the governing equation for thin plates is the same as that for plane elasticity in terms of the Airy stress function.

The afore-cited studies reveal that there is a need to investigate the stress singularities at a bi-material interface of thick plates under bending. The aim of this paper is to develop a solution for determining William's type singularities at a bi-material corner in the Mindlin plate theory [18] because this plate theory is frequently applied in solving thick plate problems. The methodology used in this work adopts the eigenfunction 
expansion method developed by Hartranft and Sih [12] for three-dimensional elasticity problems. This methodology is different from those used by Burton and Sinclair [10] and Huang [11] for isotropic and homogeneous thick plates. The numerical results are given in graphic form for the singularity orders of moments and shear forces at the interface corner in bonded dissimilar isotropic plates and at the vertex of a bi-material wedge with simply supported radial edges. Furthermore, the results for bonded dissimilar isotropic plates are compared with those of the classical plate theory. The solution developed herein is important because only after the stress singularity behavior is known can one properly account for singularities in numerical solutions of complex problems involving a bi-material interface.

\section{Governing equations}

Consider the bi-material plate shown in Fig. 1. The plate is separated into two regions. Each region is an isotropic and homogeneous plate and has its own material properties and thickness. Based on the Mindlin plate theory, the equilibrium equations with no external loading in terms of stress resultants (moments and shear forces) in polar coordinates are as follows for each region (cf. [18]):

$$
\begin{aligned}
& M_{r, r}^{(i)}+\frac{1}{r} M_{r \theta, \theta_{i}}^{(i)}+\frac{M_{r}^{(i)}-M_{\theta}^{(i)}}{r}-Q_{r}^{(i)}=0, \\
& M_{r \theta, r}^{(i)}+\frac{1}{r} M_{\theta, \theta_{i}}^{(i)}+\frac{2 M_{r \theta}^{(i)}}{r}-Q_{\theta}^{(i)}=0, \\
& Q_{r, r}^{(i)}+\frac{Q_{r}^{(i)}}{r}+\frac{1}{r} Q_{\theta, \theta_{i}}^{(i)}=0,
\end{aligned}
$$

where the subscript ", $\beta$ " refers to a partial differential with respect to independent variable $\beta$, and the superscript $(i)$ denotes region $i$ (see Fig. 1). Notably, the independent variables in circumferential direction $\theta_{i}$ are defined differently in different regions. In each region, the stress resultants are related to the transverse displacement and bending rotations by

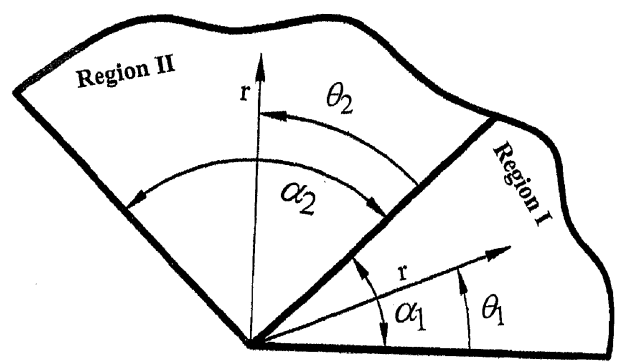

Fig. 1. A bi-material wedge with coordinates.

$$
\begin{aligned}
& M_{r}^{(i)}=-D_{i}\left[\psi_{r, r}^{(i)}+v_{i} r^{-1}\left(\psi_{r}^{(i)}+\psi_{\theta, \theta_{i}}^{(i)}\right)\right], \\
& M_{\theta}^{(i)}=-D_{i}\left[r^{-1}\left(\psi_{r}^{(i)}+\psi_{\theta, \theta_{i}}^{(i)}\right)+v_{i} \psi_{r, r}^{(i)}\right], \\
& M_{r \theta}^{(i)}=-\frac{\left(1-v_{i}\right) D_{i}}{2}\left[r^{-1}\left(\psi_{r, \theta_{i}}^{(i)}-\psi_{\theta}^{(i)}\right)+\psi_{\theta, r}^{(i)}\right], \\
& Q_{r}^{(i)}=\kappa^{2} G_{i} h_{i}\left(-\psi_{r}^{(i)}+w_{, r}^{(i)}\right), \\
& Q_{\theta}^{(i)}=\kappa^{2} G_{i} h_{i}\left(-\psi_{\theta}^{(i)}+r^{-1} w_{, \theta_{i}}^{(i)}\right),
\end{aligned}
$$

where in region $i, w^{(i)}$ is the transverse displacement of the midplane; $\psi_{r}^{(i)}$ and $\psi_{\theta}^{(i)}$ are the bending rotation of the midplane normal in the radial and circumferential directions, respectively; $h_{i}$ is the thickness of the plate; $D_{i}=E_{i} h_{i}^{3} / 12\left(1-v_{i}^{2}\right)$ is the flexural rigidity; $E_{i}$ is the modulus of elasticity; $v_{i}$ is Poisson's ratio; $\kappa^{2}$ is the shear correction factor; and $G_{i}$ is the shear modulus. The value of $\kappa^{2}$ is set equal to $\pi^{2} / 12$ [18].

Substituting Eq. (2) into Eq. (1) yields the equilibrium equations in terms of displacement components:

$$
\begin{aligned}
& \frac{D_{i}}{2}\left\{( 1 - v _ { i } ) \left(\psi_{r, r r}^{(i)}+r^{-1} \psi_{r, r}^{(i)}+r^{-2} \psi_{r, \theta_{i} \theta_{i}}^{(i)}-r^{-2} \psi_{r}^{(i)}\right.\right. \\
& \left.\quad-2 r^{-2} \psi_{\theta, \theta_{i}}^{(i)}\right)+\left(1+v_{i}\right)\left(\psi_{r, r r}^{(i)}-r^{-2} \psi_{r}^{(i)}+r^{-1} \psi_{r, r}^{(i)}\right. \\
& \left.\left.\quad-r^{-2} \psi_{\theta, \theta_{i}}^{(i)}+r^{-1} \psi_{\theta, r \theta_{i}}^{(i)}\right)\right\}+\kappa^{2} G_{i} h_{i}\left(-\psi_{r}^{(i)}+w_{, r}^{(i)}\right) \\
& \quad=0, \\
& \frac{D_{i}}{2}\left\{( 1 - v _ { i } ) \left(\psi_{\theta, r r}^{(i)}+r^{-1} \psi_{\theta, r}^{(i)}+r^{-2} \psi_{\theta, \theta_{i} \theta_{i}}^{(i)}-r^{-2} \psi_{\theta}^{(i)}\right.\right. \\
& \left.\quad+2 r^{-2} \psi_{r, \theta_{i}}^{(i)}\right)+\left(1+v_{i}\right)\left(r^{-2} \psi_{\theta, \theta_{i} \theta_{i}}^{(i)}+r^{-2} \psi_{r, \theta_{i}}^{(i)}\right. \\
& \left.\left.\quad+r^{-1} \psi_{r, r \theta_{i}}^{(i)}\right)\right\}+\kappa^{2} G_{i} h_{i}\left(-\psi_{\theta}^{(i)}+r^{-1} w_{, \theta_{i}}^{(i)}\right) \\
& \quad=0, \\
& \kappa^{2} G_{i} h_{i}\left(w_{, r r}^{(i)}+r^{-1} w_{, r}^{(i)}+r^{-2} w_{, \theta_{i} \theta_{i}}^{(i)}\right. \\
& \left.\quad-\psi_{r, r}^{(i)}-r^{-1} \psi_{r}^{(i)}-r^{-1} \psi_{\theta, \theta_{i}}^{(i)}\right)=0 .
\end{aligned}
$$

\section{Methodology}

The governing equations given in Eqs. (3a)-(3c) will be solved using an eigenfunction expansion method. The results given in the writer's previous work on isotropic and homogeneous plates [11] reveal that the singularity behaviors for moments and shears are different in the Mindlin plate theory. Consequently, they are separately considered below.

\subsection{Solution for moment singularity}

On the basis of separation of variables, the displacement components in each region for the solution of Eqs. (3a)-(3c) can be expanded in the following double series:

$\psi_{r}^{(i)}\left(r, \theta_{i}\right)=\sum_{m=0}^{\infty} \sum_{n=0,2}^{\infty} r^{\lambda_{m}+n} \Psi_{n m}^{(i)}\left(\theta_{i}, \lambda_{m}\right)$, 
$\psi_{\theta}^{(i)}\left(r, \theta_{i}\right)=\sum_{m=0}^{\infty} \sum_{n=0,2}^{\infty} r^{\lambda_{m}+n} \Phi_{n m}^{(i)}\left(\theta_{i}, \lambda_{m}\right)$,

$w^{(i)}\left(r, \theta_{i}\right)=\sum_{m=0}^{\infty} \sum_{n=0,2}^{\infty} r^{\lambda_{m}+n+1} W_{n m}^{(i)}\left(\theta_{i}, \lambda_{m}\right)$,

where the characteristic values $\lambda_{m}$ are assumed to be constants and can be complex numbers. It is noted that odd $n$ in Eqs. (4a)-(4c) will not generate any additional solution; therefore, they are not considered in Eqs. (4a) (4c).

The real part of $\lambda_{m}$ has to exceed zero to satisfy the regularity condition for finite displacement components $\left(w^{(i)}, \psi_{r}^{(i)}\right.$ and $\left.\psi_{\theta}^{(i)}\right)$ as $r$ approaches zero. Consequently, the relations between moments and displacement components given in Eq. (2) reveal that the solution in the form of Eqs. (4a)-(4c) results in moment singularities when the real part of $\lambda_{m}$ is less than one. Notably, when $\lambda_{m}$ is complex, the moments in the neighborhood of $r=0$ have the oscillatory character of the type $r^{\eta-1} \cos ($ or $\sin ) \varepsilon \log r$, where $\eta$ and $\varepsilon$ are the real part and imaginary part of $\lambda_{m}$, respectively. Nevertheless, no shear force singularities will be produced from the solution form of Eqs. (4a)(4c).

Substituting Eqs. (4a)-(4c) into Eqs. (3a)-(3c) yields

$$
\begin{aligned}
& \frac{D_{i}}{2} \sum_{m=0}^{\infty} \sum_{n=0,2}^{\infty} r^{\lambda_{m}+n-2}\left\{2\left(\lambda_{m}+n+1\right)\left(\lambda_{m}+n-1\right) \Psi_{n m}^{(i)}\right. \\
& \quad+\left(1-v_{i}\right) \Psi_{n m, \theta_{i} \theta_{i}}^{(i)}+\left[\left(1+v_{i}\right)\left(\lambda_{m}+n-1\right)\right. \\
& \left.\left.\quad-2\left(1-v_{i}\right)\right] \Phi_{n m, \theta_{i}}^{(i)}\right\}+\kappa^{2} G_{i} h_{i} \sum_{m=0}^{\infty} \sum_{n=0,2}^{\infty} r^{\lambda_{m}+n}\left[-\Psi_{n m}^{(i)}\right. \\
& \left.\quad+\left(\lambda_{m}+n+1\right) W_{n m}^{(i)}\right]=0,
\end{aligned}
$$

$$
\begin{aligned}
& \frac{D_{i}}{2} \sum_{m=0}^{\infty} \sum_{n=0,2}^{\infty} r^{\lambda_{m}+n-2}\left\{2 \Phi_{n m, \theta_{i} \theta_{i}}^{(i)}+\left(1-v_{i}\right)\left(\lambda_{m}+n+1\right)\right. \\
& \quad\left(\lambda_{m}+n-1\right) \Phi_{n m}^{(i)}+\left[\left(1+v_{i}\right)\left(\lambda_{m}+n+1\right)\right. \\
& \left.\left.\quad+2\left(1-v_{i}\right)\right] \Psi_{n m, \theta_{i}}^{(i)}\right\}+\kappa^{2} G_{i} h_{i} \sum_{m=0}^{\infty} \sum_{n=0,2}^{\infty} r^{\lambda_{m}+n}\left[-\Phi_{n m}^{(i)}\right. \\
& \left.\quad+W_{n m, \theta_{i}}^{(i)}\right]=0,
\end{aligned}
$$

$$
\begin{gathered}
\kappa^{2} G_{i} h_{i} \sum_{m=0}^{\infty} \sum_{n=0,2}^{\infty} r^{\lambda_{m}+n-1}\left[W_{n m, \theta_{i} \theta_{i}}^{(i)}+\left(\lambda_{m}+n+1\right)^{2} W_{n m}^{(i)}\right. \\
\left.-\left(\lambda_{m}+n+1\right) \Psi_{n m}^{(i)}-\Phi_{n m, \theta_{i}}^{(i)}\right]=0 .
\end{gathered}
$$

Satisfying Eqs. (5a)-(5c) leads to coefficients of $r$ with different orders equal to zero. Subsequently, a set of recurrent relationships for $W_{n m}^{(i)}, \Psi_{n m}^{(i)}$, and $\Phi_{n m}^{(i)}$ can be attained and expressed as

$$
\begin{aligned}
& \left(1-v_{i}\right) \Psi_{(n+2) m, \theta_{i} \theta_{i}}^{(i)}+2\left(\lambda_{m}+n+3\right)\left(\lambda_{m}+n+1\right) \Psi_{(n+2) m}^{(i)} \\
& \quad+\left[\left(1+v_{i}\right)\left(\lambda_{m}+n+1\right)-2\left(1-v_{i}\right)\right] \Phi_{(n+2) m, \theta_{i}}^{(i)} \\
& \quad=-\frac{2 \kappa^{2} G_{i} h_{i}}{D_{i}}\left(-\Psi_{n m}^{(i)}+\left(\lambda_{m}+n+1\right) W_{n m}^{(i)}\right), \\
& 2 \Phi_{(n+2) m, \theta_{i} \theta_{i}}^{(i)}\left(1-v_{i}\right)\left(\lambda_{m}+n+3\right)\left(\lambda_{m}+n+1\right) \Phi_{(n+2) m}^{(i)} \\
& \quad+\left[\left(1+v_{i}\right)\left(\lambda_{m}+n+3\right)+2\left(1-v_{i}\right)\right] \Psi_{(n+2) m, \theta_{i}}^{(i)} \\
& \quad=-\frac{2 \kappa^{2} G_{i} h_{i}}{D_{i}}\left(-\Phi_{n m}^{(i)}+W_{n m, \theta_{i}}^{(i)}\right), \\
& W_{(n+2) m, \theta_{i} \theta_{i}}^{(i)}+\left(\lambda_{m}+n+3\right)^{2} W_{(n+2) m}^{(i)} \\
& \quad-\left(\lambda_{m}+n+3\right) \Psi_{(n+2) m}^{(i)}-\Phi_{(n+2) m, \theta_{i}}^{(i)}=0 .
\end{aligned}
$$

Furthermore, the following equations can be established from the coefficients of the lowest order of $r$ in Eqs. (5a)-(5c):

$$
\begin{aligned}
& \left(1-v_{i}\right) \Psi_{0 m, \theta_{i} \theta_{i}}^{(i)}+2\left(\lambda_{m}+1\right)\left(\lambda_{m}-1\right) \Psi_{0 m}^{(i)} \\
& +\left[\left(1+v_{i}\right)\left(\lambda_{m}-1\right)-2\left(1-v_{i}\right)\right] \Phi_{0 m, \theta_{i}}^{(i)}=0, \\
& 2 \Phi_{0 m, \theta_{i} \theta_{i}}^{(i)}+\left(1-v_{i}\right)\left(\lambda_{m}+1\right)\left(\lambda_{m}-1\right) \Phi_{0 m}^{(i)} \\
& +\left[\left(1+v_{i}\right)\left(\lambda_{m}+1\right)+2\left(1-v_{i}\right)\right] \Psi_{0 m, \theta_{i}}^{(i)}=0, \\
& W_{0 m, \theta_{i} \theta_{i}}^{(i)}+\left(\lambda_{m}+1\right)^{2} W_{0 m}^{(i)}-\left(\lambda_{m}+1\right) \Psi_{0 m}^{(i)}-\Phi_{0 m, \theta_{i}}^{(i)}=0 .
\end{aligned}
$$

It is easy to find that the general solution for the set of linear ordinary differential equations given by Eqs. (7a)(7c) is

$$
\begin{aligned}
\Psi_{0 m}^{(i)}\left(\theta_{i}, \lambda_{m}\right)= & A_{1}^{(i)} \cos \left(\lambda_{m}+1\right) \theta_{i}+A_{2}^{(i)} \sin \left(\lambda_{m}+1\right) \theta_{i} \\
& +A_{3}^{(i)} \cos \left(\lambda_{m}-1\right) \theta_{i} \\
& +A_{4}^{(i)} \sin \left(\lambda_{m}-1\right) \theta_{i} \\
\Phi_{0 m}^{(i)}\left(\theta_{i}, \lambda_{m}\right)= & A_{2}^{(i)} \cos \left(\lambda_{m}+1\right) \theta_{i}-A_{1}^{(i)} \sin \left(\lambda_{m}+1\right) \theta_{i} \\
& +k_{i} A_{4}^{(i)} \cos \left(\lambda_{m}-1\right) \theta_{i} \\
& -k_{i} A_{3}^{(i)} \sin \left(\lambda_{m}-1\right) \theta_{i}, \\
W_{0 m}^{(i)}\left(\theta_{i}, \lambda_{m}\right)= & C_{1}^{(i)} \cos \left(\lambda_{m}+1\right) \theta_{i}+C_{2}^{(i)} \sin \left(\lambda_{m}+1\right) \theta_{i} \\
& +\gamma_{i} A_{3}^{(i)} \cos \left(\lambda_{m}-1\right) \theta_{i} \\
& +\gamma_{i} A_{4}^{(i)} \sin \left(\lambda_{m}-1\right) \theta_{i},
\end{aligned}
$$

where

$$
\begin{aligned}
& k_{i}=-\frac{3-v_{i}+\lambda_{m}+v_{i} \lambda_{m}}{3-v_{i}-\lambda_{m}-v_{i} \lambda_{m}}, \\
& \gamma_{i}=\frac{v_{i}-1}{-3+\lambda_{m}+v_{i}+v_{i} \lambda_{m}},
\end{aligned}
$$

and the characteristic value $\lambda_{m}$ and the coefficients $A_{1}^{(i)}$, $A_{2}^{(i)}, A_{3}^{(i)}, A_{4}^{(i)}, C_{1}^{(i)}$, and $C_{2}^{(i)}$ are to be determined by satisfying the radial boundary conditions and continuity conditions cross interfaces of two regions. 
The solution for $W_{n m}^{(i)}, \Psi_{n m}^{(i)}$, and $\Phi_{n m}^{(i)}$ with $n>0$ can be obtained by solving Eqs. (6a)-(6c). However, the singularity behaviors of moments are determined from $W_{n m}^{(i)}, \Psi_{n m}^{(i)}$, and $\Phi_{n m}^{(i)}$ corresponding to the lowest order of $r$, namely, $W_{0 m}^{(i)}, \Psi_{0 m}^{(i)}$, and $\Phi_{0 m}^{(i)}$; therefore, the solution for $W_{n m}^{(i)}, \Psi_{n m}^{(i)}$, and $\Phi_{n m}^{(i)}$ with $n>0$ will not be further considered here.

\subsection{Solution for shear force singularity}

From the investigations reported in [11,17], it is discovered that the displacement components in each region for the solution of Eqs. (3a)-(3c) can be expanded in the following double series to consider the shear force singularity:

$\psi_{r}^{(i)}\left(r, \theta_{i}\right)=\sum_{m=0}^{\infty} \sum_{n=0,2}^{\infty} r^{\bar{\lambda}_{m}+n+1} \bar{\Psi}_{n m}^{(i)}\left(\theta_{i}, \bar{\lambda}_{m}\right)$,

$\psi_{\theta}^{(i)}\left(r, \theta_{i}\right)=\sum_{m=0}^{\infty} \sum_{n=0,2}^{\infty} r^{\bar{\lambda}_{m}+n+1} \bar{\Phi}_{n m}^{(i)}\left(\theta_{i}, \bar{\lambda}_{m}\right)$

$w^{(i)}\left(r, \theta_{i}\right)=\sum_{m=0}^{\infty} \sum_{n=0,2}^{\infty} r^{\bar{\lambda}_{m}+n} \bar{W}_{n m}^{(i)}\left(\theta_{i}, \bar{\lambda}_{m}\right)$,

where the characteristic values $\bar{\lambda}_{m}$ are also assumed to be constants and can be complex numbers. Again, the real part of $\bar{\lambda}_{m}$ must be positive to satisfy the regularity condition under $r$ approaching zero. The relations between stress resultants and displacement components given in Eq. (2) indicate that the solution form of Eqs. (9a)-(9c) will produce a shear force singularity when $r$ approaches zero if the real part of $\bar{\lambda}_{m}$ is less than one, but no moment singularity will be given. It is also noted that when $\bar{\lambda}_{m}$ is complex the shear forces in the neighborhood of $r=0$ have the oscillatory character of the type $r^{\bar{\eta}-1} \sin ($ or $\cos ) \bar{\varepsilon} \log r$, where $\bar{\eta}$ and $\bar{\varepsilon}$ are the real part and imaginary part of $\bar{\lambda}_{m}$, respectively.

Using a procedure similar to that employed in the previous section and substituting Eqs. (9a)-(9c) into Eqs. (3a)-(3c), one finds that $\bar{W}_{n m}^{(i)}, \bar{\Psi}_{n m}^{(i)}$ and $\bar{\Phi}_{n m}^{(i)}$ have to satisfy

$$
\begin{aligned}
& \left(1-v_{i}\right) \bar{\Psi}_{(n+2) m, \theta_{i} \theta_{i}}^{(i)}+2\left(\bar{\lambda}_{m}+n+4\right)\left(\bar{\lambda}_{m}+n+2\right) \bar{\Psi}_{(n+2) m}^{(i)} \\
& +\left[\left(1+v_{i}\right)\left(\bar{\lambda}_{m}+n+2\right)-2\left(1-v_{i}\right)\right] \bar{\Phi}_{(n+2) m, \theta_{i}}^{(i)} \\
& +\frac{2 \kappa^{2} G_{i} h_{i}}{D_{i}}\left(\bar{\lambda}_{m}+n+2\right) \bar{W}_{(n+2) m}^{(i)} \\
& \quad=\frac{2 \kappa^{2} G_{i} h_{i}}{D_{i}} \bar{\Psi}_{n m}^{(i)}, \\
& 2 \bar{\Phi}_{(n+2) m, \theta_{i} \theta_{i}}^{(i)}\left(1-v_{i}\right)\left(\bar{\lambda}_{m}+n+4\right)\left(\bar{\lambda}_{m}+n+2\right) \bar{\Phi}_{(n+2) m}^{(i)} \\
& +\left[\left(1+v_{i}\right)\left(\bar{\lambda}_{m}+n+4\right)+2\left(1-v_{i}\right)\right] \bar{\Psi}_{(n+2) m, \theta_{i}}^{(i)} \\
& +\frac{2 \kappa^{2} G_{i} h_{i}}{D_{i}} \bar{W}_{(n+2) m, \theta_{i}}^{(i)} \\
& \quad=\frac{2 \kappa^{2} G_{i} h_{i}}{D_{i}} \bar{\Phi}_{n m}^{(i)},
\end{aligned}
$$

$$
\begin{gathered}
\bar{W}_{(n+2) m, \theta_{i} \theta_{i}}^{(i)}+\left(\bar{\lambda}_{m}+n+2\right)^{2} \bar{W}_{(n+2) m}^{(i)} \\
=\left(\bar{\lambda}_{m}+n+2\right) \bar{\Psi}_{n m}^{(i)}+\bar{\Phi}_{n m, \theta_{i}}^{(i)},
\end{gathered}
$$

and

$$
\begin{aligned}
& \left(1-v_{i}\right) \bar{\Psi}_{0 m, \theta_{i} \theta_{i}}^{(i)}+2 \bar{\lambda}_{m}\left(\bar{\lambda}_{m}+2\right) \bar{\Psi}_{0 m}^{(i)}+\left[\left(1+v_{i}\right) \bar{\lambda}_{m}\right. \\
& \left.\quad-2\left(1-v_{i}\right)\right] \bar{\Phi}_{0 m, \theta_{i}}^{(i)}+\frac{2 \kappa^{2} G_{i} h_{i}}{D_{i}} \bar{\lambda}_{m} \bar{W}_{0 m}^{(i)}=0 \\
& 2 \bar{\Phi}_{0 m, \theta_{i} \theta_{i}}^{(i)}+\left(1-v_{i}\right) \bar{\lambda}_{m}\left(\bar{\lambda}_{m}+2\right) \bar{\Phi}_{0 m}^{(i)}+\left[\left(1+v_{i}\right)\left(\bar{\lambda}_{m}+2\right)\right. \\
& \left.\quad+2\left(1-v_{i}\right)\right] \bar{\Psi}_{0 m, \theta_{i}}^{(i)}+\frac{2 \kappa^{2} G_{i} h_{i}}{D_{i}} \bar{W}_{0 m, \theta_{i}}^{(i)}=0 \\
& \bar{W}_{0 m, \theta_{i} \theta_{i}}^{(i)}+\bar{\lambda}_{m}^{2} \bar{W}_{0 m}^{(i)}=0 .
\end{aligned}
$$

Again, to investigate the shear force singularity, one only needs to consider the solution for $\bar{W}_{0 m}^{(i)}, \bar{\Psi}_{0 m}^{(i)}$ and $\bar{\Phi}_{0 m}^{(i)}$. By employing a typical mathematical procedure for solving a set of linear ordinary differential equations, one is able to find the general solution of Eqs. (11a) (11c):

$$
\begin{aligned}
\bar{\Psi}_{0 m}^{(i)}\left(\theta_{i}, \bar{\lambda}_{m}\right)= & \bar{A}_{1}^{(i)} \cos \bar{\lambda}_{m} \theta_{i}+\bar{A}_{2}^{(i)} \sin \bar{\lambda}_{m} \theta_{i} \\
& +\bar{A}_{3}^{(i)} \cos \left(\bar{\lambda}_{m}+2\right) \theta_{i}+\bar{A}_{4}^{(i)} \sin \left(\bar{\lambda}_{m}+2\right) \theta_{i}, \\
\bar{\Phi}_{0 m}^{(i)}\left(\theta_{i}, \bar{\lambda}_{m}\right)= & \bar{B}_{1}^{(i)} \cos \bar{\lambda}_{m} \theta_{i}+\bar{B}_{2}^{(i)} \sin \bar{\lambda}_{m} \theta_{i} \\
& +\bar{A}_{4}^{(i)} \cos \left(\bar{\lambda}_{m}+2\right) \theta_{i}-\bar{A}_{3}^{(i)} \sin \left(\bar{\lambda}_{m}+2\right) \theta_{i}, \\
\bar{W}_{0 m}^{(i)}\left(\theta_{i}, \bar{\lambda}_{m}\right)= & \bar{l}_{1}^{(i)}\left(\bar{A}_{1}^{(i)} \cos \bar{\lambda}_{m} \theta_{i}+\bar{A}_{2}^{(i)} \sin \bar{\lambda}_{m} \theta_{i}\right) \\
& +\bar{l}_{2}^{(i)}\left(\bar{B}_{2}^{(i)} \cos \bar{\lambda}_{m} \theta_{i}-\bar{B}_{1}^{(i)} \sin \bar{\lambda}_{m} \theta_{i}\right),
\end{aligned}
$$

where

$\bar{l}_{1}^{(i)}=\frac{-D_{i}}{2 \kappa^{2} G_{i} h_{i}}\left(3-v_{i}+\left(1+v_{i}\right)\left(1+\bar{\lambda}_{m}\right)\right)$,

and

$\bar{l}_{2}^{(i)}=\frac{D_{i}}{2 \kappa^{2} G_{i} h_{i}}\left(2\left(1-v_{i}\right)-\left(1+v_{i}\right) \bar{\lambda}_{m}\right)$.

The characteristic value $\bar{\lambda}_{m}$ and the coefficients $\bar{A}_{1}^{(i)}, \bar{A}_{2}^{(i)}$, $\bar{A}_{3}^{(i)}, \bar{A}_{4}^{(i)}, \bar{B}_{1}^{(i)}$, and $\bar{B}_{2}^{(i)}$ are to be determined by satisfying the radial boundary conditions and continuity conditions.

\section{Case study}

As a demonstration of the procedure for determining the characteristic values $\lambda_{m}$ and $\bar{\lambda}_{m}$, two examples will be given: in one, singularities at the interface joint in bonded dissimilar isotropic plates are considered; in the other, singularities at the vertex of a bi-material wedge with simply supported radial edges are investigated. The effects of the material and geometric properties of the plates on $\lambda_{m}$ and $\bar{\lambda}_{m}$ will be discussed. 


\subsection{Case I: Bonded dissimilar isotropic plates}

Consider a plate composed of two isotropic materials perfectly bonded together as shown in Fig. 2. The plate is separated into two regions, each of which has its own material properties and thickness. The solutions given in Eqs. (8a)-(8c) and Eqs. (12a)-(12c) for moment singularity and shear force singularity, respectively, have to satisfy the continuity conditions along the two interfaces.

The continuity conditions are expressed in terms of the displacement components, moments, and shear force along the interfaces (see Fig. 2):

$$
\begin{aligned}
& \left.w^{(1)}\left(r, \theta_{1}\right)\right|_{\theta_{1}= \pm \alpha_{1} / 2}=\left.w^{(2)}\left(r, \theta_{2}\right)\right|_{\theta_{2}=\mp \alpha_{2} / 2}, \\
& \left.\psi_{r}^{(1)}\left(r, \theta_{1}\right)\right|_{\theta_{1}= \pm \alpha_{1} / 2}=\left.\psi_{r}^{(2)}\left(r, \theta_{2}\right)\right|_{\theta_{2}=\mp \alpha_{2} / 2}, \\
& \left.\psi_{\theta}^{(1)}\left(r, \theta_{1}\right)\right|_{\theta_{1}= \pm \alpha_{1} / 2}=\left.\psi_{\theta}^{(2)}\left(r, \theta_{2}\right)\right|_{\theta_{2}=\mp \alpha_{2} / 2}, \\
& \left.M_{\theta}^{(1)}\left(r, \theta_{1}\right)\right|_{\theta_{1}= \pm \alpha_{1} / 2}=\left.M_{\theta}^{(2)}\left(r, \theta_{2}\right)\right|_{\theta_{2}=\mp \alpha_{2} / 2}, \\
& \left.M_{r \theta}^{(1)}\left(r, \theta_{1}\right)\right|_{\theta_{1}= \pm \alpha_{1} / 2}=\left.M_{r \theta}^{(2)}\left(r, \theta_{2}\right)\right|_{\theta_{2}=\mp \alpha_{2} / 2}, \\
& \left.Q_{\theta}^{(1)}\left(r, \theta_{1}\right)\right|_{\theta_{1}= \pm \alpha_{1} / 2}=\left.Q_{\theta}^{(2)}\left(r, \theta_{2}\right)\right|_{\theta_{2}=\mp \alpha_{2} / 2} .
\end{aligned}
$$

Because of the symmetry of the domain and continuity conditions considered here, Eqs. (8a)-(8c) and Eqs. (12a)-(12c) can be divided into symmetric and antisymmetric deformation parts, respectively, both of which must satisfy Eqs. (13a)-(13f).

\subsubsection{Characteristic equation for moment singularity}

Substituting the symmetric deformation part in Eqs. (8a)-(8c) into Eqs. (13a)-(13f) yields six linear independent homogeneous algebraic equations for the co-

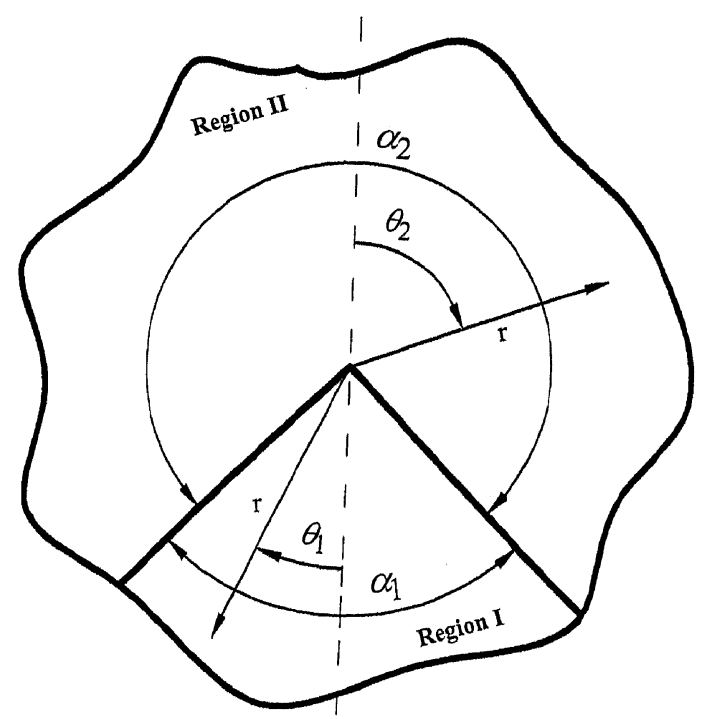

Fig. 2. Interface corner in bonded dissimilar materials. efficients $A_{1}^{(i)}, A_{3}^{(i)}$, and $C_{1}^{(i)}$ with $i=1$ and 2 . This results in a six by six determinant equal to zero, which is necessary to have a non-trivial solution for these coefficients. With the aid of the symbolic logic software program MATHEMATICA, the zero determinant results in $\lambda_{m}$ satisfying

$$
\begin{aligned}
& \left\{\kappa^{2} G_{1} h_{1} \cos \left(\left(\lambda_{m}+1\right) \alpha_{2} / 2\right) \sin \left(\left(\lambda_{m}+1\right) \alpha_{1} / 2\right)\right. \\
& \left.\quad+\kappa^{2} G_{2} h_{2} \cos \left(\left(\lambda_{m}+1\right) \alpha_{1} / 2\right) \sin \left(\left(\lambda_{m}+1\right) \alpha_{2} / 2\right)\right\}=0,
\end{aligned}
$$

or

$\left\{\delta_{1} \sin \lambda_{m} \alpha_{1} \sin \lambda_{m} \alpha_{2}+\delta_{2} \sin \lambda_{m} \alpha_{1} \sin \alpha_{2}\right.$

$$
\begin{aligned}
& +\delta_{3} \sin \alpha_{1} \sin \lambda_{m} \alpha_{2}+\delta_{4} \sin \alpha_{1} \sin \alpha_{2} \\
& \left.+\delta_{5} \cos \lambda_{m} \alpha_{1} \cos \lambda_{m} \alpha_{2}-\delta_{5} \cos \alpha_{1} \cos \alpha_{2}\right\}=0,
\end{aligned}
$$

where

$$
\begin{aligned}
\delta_{1}= & D_{1}^{2}\left(-1+v_{1}\right)^{2}\left(1+v_{1}\right)\left(-3+v_{2}\right) \\
& -2 D_{1} D_{2}\left(-1+v_{1}\right)^{2}\left(-1+v_{2}\right)^{2} \\
& +D_{2}^{2}\left(-3+v_{1}\right)\left(-1+v_{2}\right)^{2}\left(1+v_{2}\right), \\
\delta_{2}= & \lambda_{m}\left(1+v_{2}\right)\left[D_{1}^{2}\left(-1+v_{1}\right)^{2}\left(1+v_{1}\right)\right. \\
& -2 D_{1} D_{2}\left(-1+v_{1}\right)^{2}\left(-1+v_{2}\right) \\
& \left.+D_{2}^{2}\left(-3+v_{1}\right)\left(-1+v_{2}\right)^{2}\right], \\
\delta_{3}= & \lambda_{m}\left(1+v_{1}\right)\left[D_{1}^{2}\left(-1+v_{1}\right)^{2}\left(-3+v_{2}\right)\right. \\
& -2 D_{1} D_{2}\left(-1+v_{1}\right)\left(-1+v_{2}\right)^{2} \\
& \left.+D_{2}^{2}\left(1+v_{2}\right)\left(-1+v_{2}\right)^{2}\right], \\
\delta_{4}= & D_{1}^{2} \lambda_{m}^{2}\left(-1+v_{1}\right)^{2}\left(1+v_{1}\right)\left(1+v_{2}\right) \\
& -2 D_{1} D_{2}\left(-1+v_{1}\right)\left(-1+v_{2}\right)\left(-4+\lambda_{m}^{2}\left(1+v_{1}\right)\right. \\
& \left.\times\left(1+v_{2}\right)\right)+D_{2}^{2} \lambda_{m}^{2}\left(1+v_{1}\right)\left(-1+v_{2}\right)^{2}\left(1+v_{2}\right),
\end{aligned}
$$

$\delta_{5}=8 D_{1} D_{2}\left(-1+v_{1}\right)\left(-1+v_{2}\right)$.

It should be noted that the situation where $\lambda_{m}$ does not satisfy Eq. (14b) but only satisfies Eq. (14a) causes the coefficients $A_{1}^{(i)}$ and $A_{3}^{(i)}$ to vanish. The solution given in Eqs. (4a)-(4c) degenerates to the solution given by Eqs. (9a)-(9c), and no moment singularity will occur. Consequently, Eq. (14a) is abandoned in this case, and Eq. (14b) is the characteristic equation.

To verify the correctness of Eq. (14b), let us consider a special case in which $D_{1} / D_{2}$ approaches zero and compare the resulting characteristic equations with published ones. As $D_{1} / D_{2}$ approaches zero, the characteristic equation (14b) becomes

$$
\begin{aligned}
& \left\{\left(-3+v_{2}\right) \sin \lambda_{m} \alpha_{2}+\lambda_{m}\left(1+v_{2}\right) \sin \alpha_{2}\right\} \\
& \quad \times\left\{\sin \lambda_{m} \alpha_{1}+\lambda_{m} \sin \alpha_{1}\right\}=0 .
\end{aligned}
$$

Physically, when $D_{1} / D_{2}$ approaches zero, region I (see Fig. 2) reacts just like a wedge with two radial clamped edges, and region II reacts like a wedge with two free 

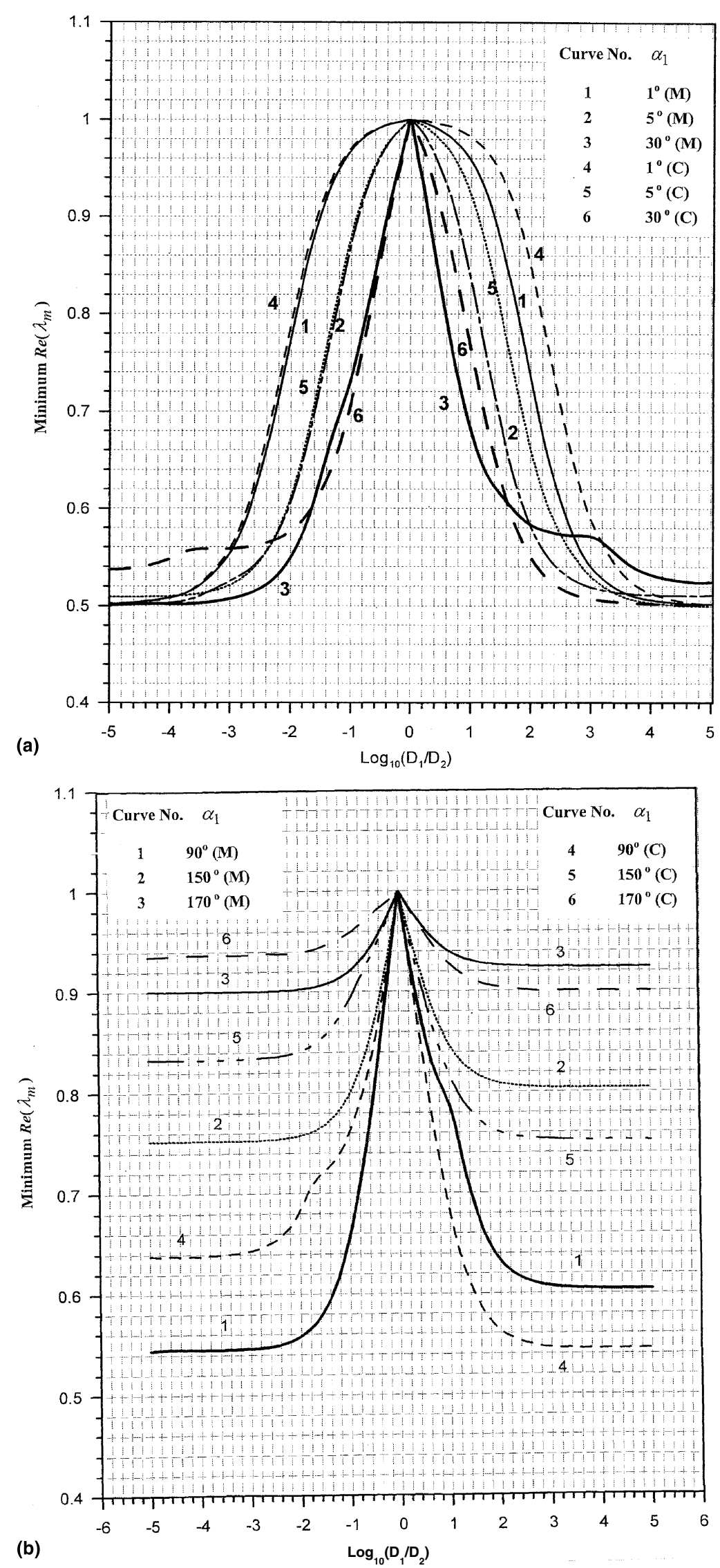

Fig. 3. Variation of the minimum $\operatorname{Re}\left(\lambda_{m}\right)$ with $D_{1} / D_{2}$ for: (a) $\alpha_{1}=1^{\circ}, 5^{\circ}$, and $30^{\circ}$; (b) $\alpha_{1}=90^{\circ}, 150^{\circ}$ and $170^{\circ}$. 
radial edges. Carefully examining Eq. (16) and the results of Burton and Sinclair [10] and Huang [11] reveals that

$\left(-3+v_{2}\right) \sin \lambda_{m} \alpha_{2}+\lambda_{m}\left(1+v_{2}\right) \sin \alpha_{2}=0$

and

$\sin \lambda_{m} \alpha_{1}+\lambda_{m} \sin \alpha_{1}=0$

are, respectively, the characteristic equation for a wedge with two free radial edges and that for a wedge with two clamped radial edges in the case of symmetric deformation.

Similarly, by substituting the anti-symmetric deformation part in Eqs. (8a)-(8c) into Eqs. (13a)-(13f) and performing lengthy mathematical operations, one finally obtains the characteristic equation

$$
\begin{aligned}
& -\delta_{1} \sin \lambda_{m} \alpha_{1} \sin \lambda_{m} \alpha_{2}+\delta_{2} \sin \lambda_{m} \alpha_{1} \sin \alpha_{2} \\
& \quad+\delta_{3} \sin \alpha_{1} \sin \lambda_{m} \alpha_{2}-\delta_{4} \sin \alpha_{1} \sin \alpha_{2} \\
& \quad-\delta_{5} \cos \lambda_{m} \alpha_{1} \cos \lambda_{m} \alpha_{2}+\delta_{5} \cos \alpha_{1} \cos \alpha_{2}=0 .
\end{aligned}
$$

Apparently, the characteristic values $\lambda_{m}$ for Eqs. (14a), and (17) are dependent on $D_{1} / D_{2}, v_{1}, v_{2}$, and $\alpha_{1}$ (or $\alpha_{2}$ ). Under the restriction that the real part of $\lambda_{m}\left(\operatorname{Re}\left(\lambda_{m}\right)\right)$ exceeds zero, the variation of the minimum $\operatorname{Re}\left(\lambda_{m}\right)$ with $D_{1} / D_{2}$ for $v_{1}=v_{2}=0.3$ is shown in Figs. 3(a) and (b), in which (M) and (C), respectively, denote the results for the Mindlin plate theory and classical plate theory. Fig. 4 depicts the effect of Poisson's ratios on the solution for $\lambda_{m}$. Notably, when $v_{1}=v_{2}$, the minimum $\operatorname{Re}\left(\lambda_{m}\right)$ for $D_{1} / D_{2}=\chi_{1}$ and $\alpha_{1}=\chi_{2}$ is the same as that for $D_{1} / D_{2}=1 / \chi_{1}$ and $\alpha_{2}=\chi_{2}$, where $\chi_{1}$ and $\chi_{2}$ are physically acceptable arbitrary constants. Similarly, if regions I and II have the same Young's modulus and plate thickness, then the minimum $\operatorname{Re}\left(\lambda_{m}\right)$ for $\left(v_{1}, v_{2}\right)=\left(\chi_{3}, \chi_{4}\right)$ and $\alpha_{1}=\chi_{2}$ will be equal to that for $\left(v_{1}, v_{2}\right)=\left(\chi_{4}, \chi_{3}\right)$ and $\alpha_{2}=\chi_{2}$, where $\chi_{3}$ and $\chi_{4}$ are also physically acceptable arbitrary constants.

Recalling that $\operatorname{Re}\left(\lambda_{m}\right)<1$ results in a moment singularity when $r$ approaches zero, several facts are observed from Figs. 3(a) and (b), and Fig. 4. When $D_{1} \neq D_{2}$, the moment singularity always occurs for all values of $\alpha_{1}$ considered in Fig. 3(a) and (b). Nevertheless, it should be noted that there will not be singularity for $\alpha_{1}=0^{\circ}, 180^{\circ}$ or $360^{\circ}$. The singularity generally becomes stronger as $D_{1} / D_{2}$ moves further away from 1 . The singularity caused by unequal Poisson's ratios alone is rather weak.

To compare the present results with those from the classical plate theory, Figs. 3(a) and (b) also depict the results for the classical plate theory obtained using William's approach [19]. The comparison shows that when $\alpha_{1} \geqslant 90^{\circ}$, the singularity is stronger for the Mindlin plate theory when $D_{1} / D_{2}$ is less than one, while the opposite situation is observed when $D_{1} / D_{2}$ exceeds

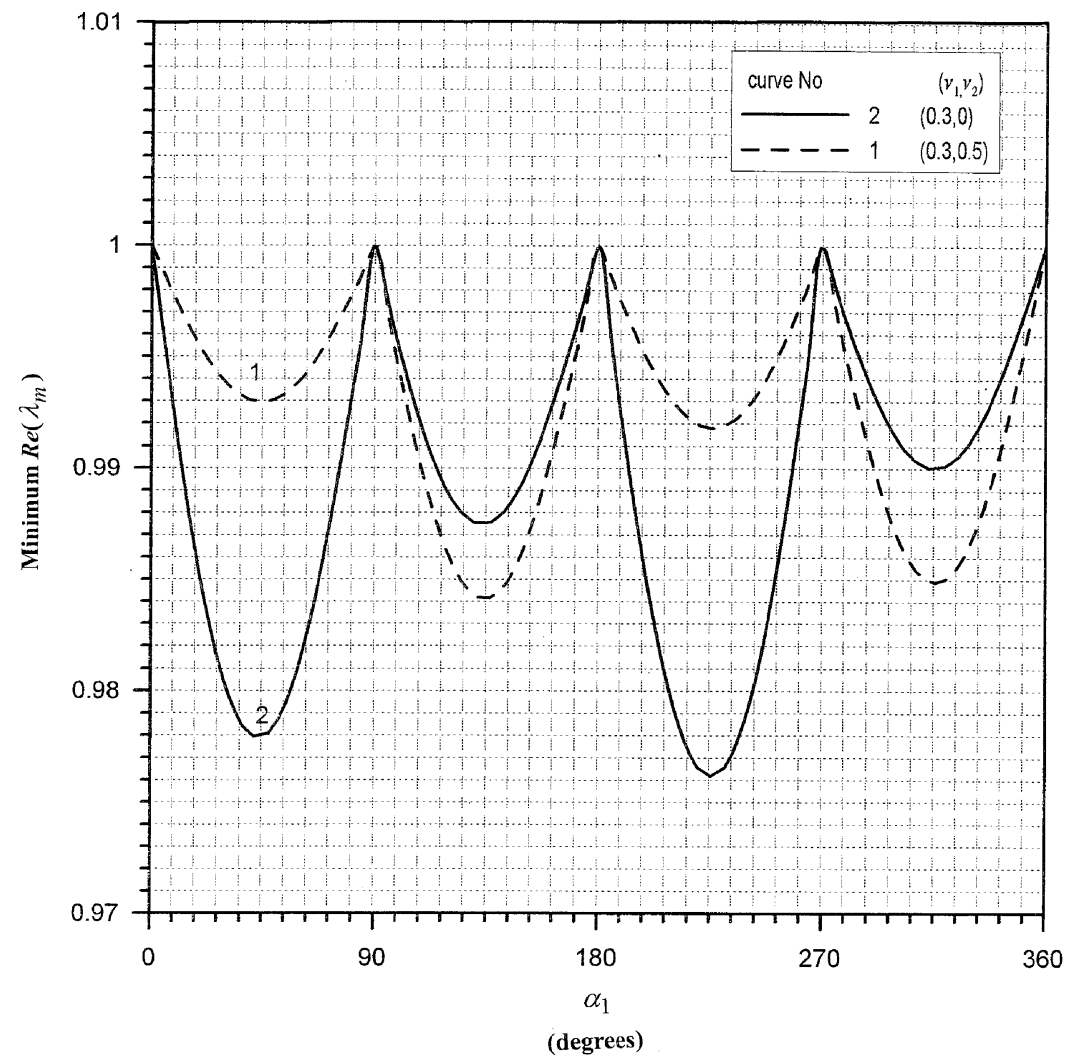

Fig. 4. Variation of the minimum $\operatorname{Re}\left(\lambda_{m}\right)$ with $\alpha_{1}$ for $E_{1}=E_{2}$ and $h_{1}=h_{2}$. 
one. For small $\alpha_{1}$ (say $\leqslant 5^{\circ}$ ), the two theories produce very similar singularities for $D_{1} / D_{2}$ less than one, while the Mindlin theory produces much stronger singularity than the classical theory does for $D_{1} / D_{2}$ between 1 and 1000 .

4.1.2. Characteristic equation for shear force singularity

Substituting the symmetric deformation part in Eqs. (12a)-(12c) into Eqs. (13a)-(13f) and performing the procedure employed in the previous section, one is able to obtain the following characteristic equations for $\bar{\lambda}_{m}$ :

$$
\begin{aligned}
& G_{1} h_{1} \cos \left(\bar{\lambda}_{m} \alpha_{2} / 2\right) \sin \left(\bar{\lambda}_{m} \alpha_{1} / 2\right) \\
& \quad+G_{2} h_{2} \cos \left(\bar{\lambda}_{m} \alpha_{1} / 2\right) \sin \left(\bar{\lambda}_{m} \alpha_{2} / 2\right)=0
\end{aligned}
$$

for symmetric deformation and

$$
\begin{aligned}
& G_{1} h_{1} \cos \left(\bar{\lambda}_{m} \alpha_{1} / 2\right) \sin \left(\bar{\lambda}_{m} \alpha_{2} / 2\right) \\
& \quad+G_{2} h_{2} \cos \left(\bar{\lambda}_{m} \alpha_{2} / 2\right) \sin \left(\bar{\lambda}_{m} \alpha_{1} / 2\right)=0
\end{aligned}
$$

for anti-symmetric deformation. When $G_{1} h_{1} / G_{2} h_{2}$ approaches zero, the two equations can also be reduced to the characteristics equations for an isotropic and homogeneous wedge with clamped radial edges or free radial edges [11]. Obviously, the resulting characteristic value is dependent on $G_{1} h_{1} / G_{2} h_{2}$ and $\alpha_{1}$ (or $\alpha_{2}$ ) but is independent of Poisson's ratios.

Fig. 5 depicts the variation of the minimum $\operatorname{Re}\left(\bar{\lambda}_{m}\right)$ with $G_{1} h_{1} / G_{2} h_{2}$ for several values of $\alpha_{1}$. Some observations can be made from this figure. When $G_{1} h_{1} \neq G_{2} h_{2}$, the shear force singularity always occurs for all values of $\alpha_{1}$ considered in this figure. However, it should be noted that no singularity will occur for $\alpha_{1}=0^{\circ}, 180^{\circ}$ or $360^{\circ}$. The minimum $\operatorname{Re}\left(\lambda_{m}\right)$ for $G_{1} h_{1} / G_{2} h_{2}=y_{1}$ and $\alpha_{1}=y_{2}$ will be the same as that for $G_{1} h_{1} / G_{2} h_{2}=1 / y_{1}$ and $\alpha_{2}=y_{2}$, where $y_{1}$ and $y_{2}$ are physically acceptable arbitrary constants. Hence, only some values less than $180^{\circ}$ for $\alpha_{1}$ are considered in this figure. The singularity becomes stronger as $G_{1} h_{1} / G_{2} h_{2}$ moves further away from 1. For very large or small $G_{1} h_{1} / G_{2} h_{2}$ (say, $G_{1} h_{1} / G_{2} h_{2}>1000$ or $\left.G_{1} h_{1} / G_{2} h_{2}<0.001\right)$, the singularity becomes stronger as $\alpha_{1}$ becomes smaller.

\subsection{Case 2: A bi-material wedge with simply supported radial edges}

Consider a bi-material wedge as shown in Fig. 1 with simply supported boundary conditions at $\theta_{1}=0$ and $\theta_{2}=\alpha_{2}$. The radial boundary conditions are $w^{(i)}=$ $\psi_{r}^{(i)}=M_{\theta}^{(i)}=0$. The interface is perfectly bonded, and

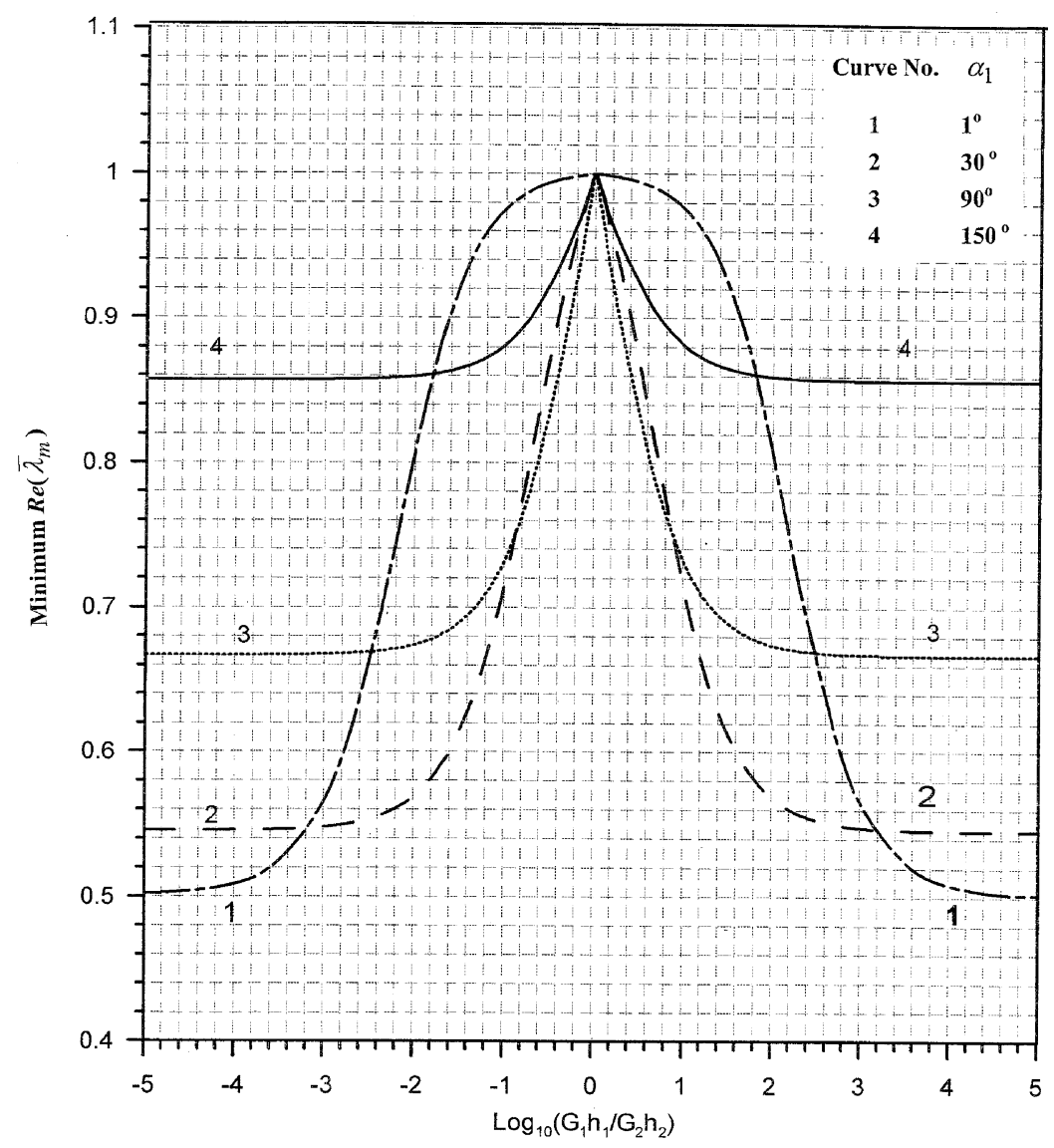

Fig. 5. Variation of the minimum $\operatorname{Re}\left(\bar{\lambda}_{m}\right)$ with $G_{1} h_{1} / G_{2} h_{2}$ for bonded dissimilar isotropic plates. 
the continuity conditions along the interface are the same as those given in Eqs. (13a)-(13f) except that these conditions are prescribed at $\theta_{1}=\alpha_{1}$ and $\theta_{2}=0$.

\subsubsection{Characteristic equation for moment singularity}

Substituting the solution given in Eqs. (8a)-(8c) into the boundary conditions and the continuity conditions yields 12 linear independent homogeneous algebraic equations for the coefficients $A_{1}^{(i)}, A_{2}^{(i)}, A_{3}^{(i)}, A_{4}^{(i)}, C_{1}^{(i)}$ and $C_{2}^{(i)}$ with $i=1$ and 2 . This results in a twelve by twelve determinant equal to zero, which is necessary to have a non-trivial solution for these coefficients. By using the symbolic logic software program MATHEMATICA, one is able to obtain the following characteristic equation for $\lambda_{m}$ that will produce moment singularities:

$$
\begin{aligned}
\xi_{1} & \sin \left(\lambda_{m}+1\right) \alpha_{2} \cos \left(\lambda_{m}-1\right) \alpha_{2} \cos \left(\lambda_{m}+1\right) \alpha_{1} \\
& \times \sin \left(\lambda_{m}-1\right) \alpha_{1}+\xi_{2} \sin \left(\lambda_{m}+1\right) \alpha_{2} \sin \left(\lambda_{m}-1\right) \alpha_{2} \\
& \times \cos \left(\lambda_{m}+1\right) \alpha_{1} \cos \left(\lambda_{m}-1\right) \alpha_{1}+\xi_{3} \sin \left(\lambda_{m}+1\right) \alpha_{2} \\
& \times \cos \left(\lambda_{m}-1\right) \alpha_{2} \sin \left(\lambda_{m}+1\right) \alpha_{1} \cos \left(\lambda_{m}-1\right) \alpha_{1} \\
& +\xi_{2} \cos \left(\lambda_{m}+1\right) \alpha_{2} \cos \left(\lambda_{m}-1\right) \alpha_{2} \sin \left(\lambda_{m}+1\right) \alpha_{1} \\
& \times \sin \left(\lambda_{m}-1\right) \alpha_{1}+\xi_{4} \cos \left(\lambda_{m}+1\right) \alpha_{2} \sin \left(\lambda_{m}-1\right) \alpha_{2} \\
& \times \cos \left(\lambda_{m}+1\right) \alpha_{1} \sin \left(\lambda_{m}-1\right) \alpha_{1}+\xi_{5} \cos \left(\lambda_{m}+1\right) \alpha_{2} \\
& \times \sin \left(\lambda_{m}-1\right) \alpha_{2} \sin \left(\lambda_{m}+1\right) \alpha_{1} \cos \left(\lambda_{m}-1\right) \alpha_{1}=0,
\end{aligned}
$$

where

$$
\begin{aligned}
\xi_{1}= & -\left[D_{1}\left(1+\lambda_{m}\right)\left(-1+v_{1}^{2}\right)-D_{2}\left(-3+\lambda_{m}+v_{1}\right.\right. \\
& \left.\left.+\lambda_{m} v_{1}\right)\left(-1+v_{2}\right)\right] \cdot\left[D _ { 1 } ( - 1 + v _ { 1 } ) \left(3+\lambda_{m}\right.\right. \\
& \left.\left.-v_{2}+\lambda_{m} v_{2}\right)-D_{2}\left(-1+\lambda_{m}\right)\left(-1+v_{2}^{2}\right)\right], \\
\xi_{2}= & -16 D_{1} D_{2}\left(-1+v_{1}\right)\left(-1+v_{2}\right), \\
\xi_{3}= & \left(-1+\lambda_{m}\right)\left[D_{1}\left(-1+v_{1}\right)+D_{2}\left(1-v_{2}\right)\right] \\
& \times\left[D_{1}\left(-1+v_{1}^{2}\right)\left(3+\lambda_{m}-v_{2}+\lambda_{m} v_{2}\right)\right. \\
& \left.-D_{2}\left(3+\lambda_{m}-v_{1}+\lambda_{m} v_{1}\right)\left(-1+v_{2}^{2}\right)\right], \\
\xi_{4}= & \left(1+\lambda_{m}\right)\left[D_{1}\left(-1+v_{1}\right)+D_{2}\left(1-v_{2}\right)\right] \\
& \times\left[D_{1}\left(-1+v_{1}^{2}\right)\left(-3+\lambda_{m}+v_{2}+\lambda_{m} v_{2}\right)\right. \\
& \left.-D_{2}\left(-3+\lambda_{m}+v_{1}+\lambda_{m} v_{1}\right)\left(-1+v_{2}^{2}\right)\right], \\
\xi_{5}= & -\left[D_{1}\left(-1+\lambda_{m}\right)\left(-1+v_{1}^{2}\right)-D_{2}\left(3+\lambda_{m}-v_{1}\right.\right. \\
& \left.\left.+\lambda_{m} v_{1}\right)\left(-1+v_{2}\right)\right] \cdot\left[D _ { 1 } ( - 1 + v _ { 1 } ) \left(-3+\lambda_{m}\right.\right. \\
& \left.\left.+v_{2}+\lambda_{m} v_{2}\right)-D_{2}\left(1+\lambda_{m}\right)\left(-1+v_{2}^{2}\right)\right] .
\end{aligned}
$$

Apparently, the characteristic values $\lambda_{m}$ for Eq. (19) are dependent on $D_{1} / D_{2}, v_{1}, v_{2}$, and $\alpha_{1}\left(\right.$ or $\left.\alpha_{2}\right)$.

To confirm the correctness of the characteristic equation, let us consider some special cases for the material properties. When $D_{1} / D_{2}$ approaches zero, one is able to reduce Eqs. (19) and (20a)-(20e) to the following equations through lengthy mathematical operations: $\sin 2 \lambda_{m} \alpha_{2}-\lambda_{m} \sin 2 \alpha_{2}=0$,

or

$\left(-3+v_{1}\right) \sin 2 \lambda_{m} \alpha_{1}-\lambda_{m}\left(1+v_{1}\right) \sin 2 \alpha_{1}=0$.

Eq. (21a) happens to be the characteristic equation for region II (see Fig. 1) with simply supported at one radial edge and free at the other radial edge [10,11]. Eq. (21b) is also the characteristic equation for region I with simply supported at one radial edge and clamped at the other radial edge [10,11]. These results are also expected from the physical sense for $D_{1} / D_{2}$ approaching zero. Similarly, by letting $D_{2} / D_{1}$ approach zero, one can also reduce Eqs. (19) and (20a)-(20e) to the characteristic equation for region I with simply supported and free radial edges and to the characteristic equation for region II with simply supported and clamped radial edges. Furthermore, by letting $D_{1}=D_{2}$ and $v_{1}=v_{2}$, Eqs. (19) and (20a)-(20e) can be reduced to the characteristic equation for an isotropic and homogeneous wedge with simply supported edges as given in [10,11].

Figs. 6 and 7, respectively, show the minimum positive real part of $\lambda_{m}$ for two general cases, which commonly occur when dissimilar materials are jointed. The first case is for the most favorable angle for the interface when two dissimilar materials are joined to give a half-plane $\left(\alpha_{1}+\alpha_{2}=180^{\circ}\right)$. The second case is for $\alpha_{2}=180^{\circ}$. Since Poisson's ratios do not considerably affect $\lambda_{m}$, the results shown in Figs. 6 and 7 are for $v_{1}=v_{2}=0.3$.

It should also be noted that in the case of $\alpha_{1}+\alpha_{2}=180^{\circ}$, the minimum $\operatorname{Re}\left(\lambda_{m}\right)$ for $D_{1} / D_{2}=\chi_{1}$ and $\alpha_{1}=\chi_{2}$ is the same as that for $D_{1} / D_{2}=1 / \chi_{1}$ and $\alpha_{2}=\chi_{2}$, where $\chi_{1}$ and $\chi_{2}$ are physically acceptable arbitrary constants. Hence, Figs. 6(a) and (b) only depict the numerical results for some values of $\alpha_{1}$ less than $90^{\circ}$. The results shown in Fig. 6(a) indicate that for very large or small $D_{1} / D_{2}$ (say, $D_{1} / D_{2} \geqslant 10^{5}$ or $D_{1} / D_{2} \leqslant 10^{-4}$ ), the singularity becomes stronger as $\alpha_{1}$ becomes smaller. There is no singularity for $\alpha_{1}=5^{\circ}$ and $D_{1} / D_{2}$ between 1 and 2000, and no singularity for $\alpha_{1}=30^{\circ}$ and $D_{1} / D_{2}$ between 1 and 10 . It is also observed that there is no singularity for $D_{1} / D_{2} \leqslant 1$ and $\alpha_{1}=75^{\circ}$.

To further investigate the effect of $\alpha_{1}$ on the singularity for the case of $\alpha_{1}+\alpha_{2}=180^{\circ}$, Fig. 6(b) shows the variation of the minimum $\operatorname{Re}\left(\lambda_{m}\right)$ with $\alpha_{1}$ for $D_{1} / D_{2}=10^{-4}, 10^{-2}, 0.1,10,100$, and $10^{4}$ when $\alpha_{1}$ is less than $90^{\circ}$. For the values of $D_{1} / D_{2}$ considered here, there is always a region for $\alpha_{1}$, where no moment singularity exists. When $D_{1} / D_{2} \leqslant 0.1$, no moment singularity exists when $\alpha_{1}$ is larger than approximately $51^{\circ}$. When $D_{1} / D_{2}=10$ and 100 , there are no singularities for $\alpha_{1} \leqslant 30^{\circ}$ and $15^{\circ}$, respectively, and there is no singularity for $D_{1} / D_{2}=10000$ and $\alpha_{1} \leqslant 2^{\circ}$.

Fig. 7 depicts the variation of the minimum $\operatorname{Re}\left(\lambda_{m}\right)$ with $D_{1} / D_{2}$ for $\alpha_{1}=30^{\circ}, 60^{\circ}, 90^{\circ}, 120^{\circ}$, and $150^{\circ}$, when 

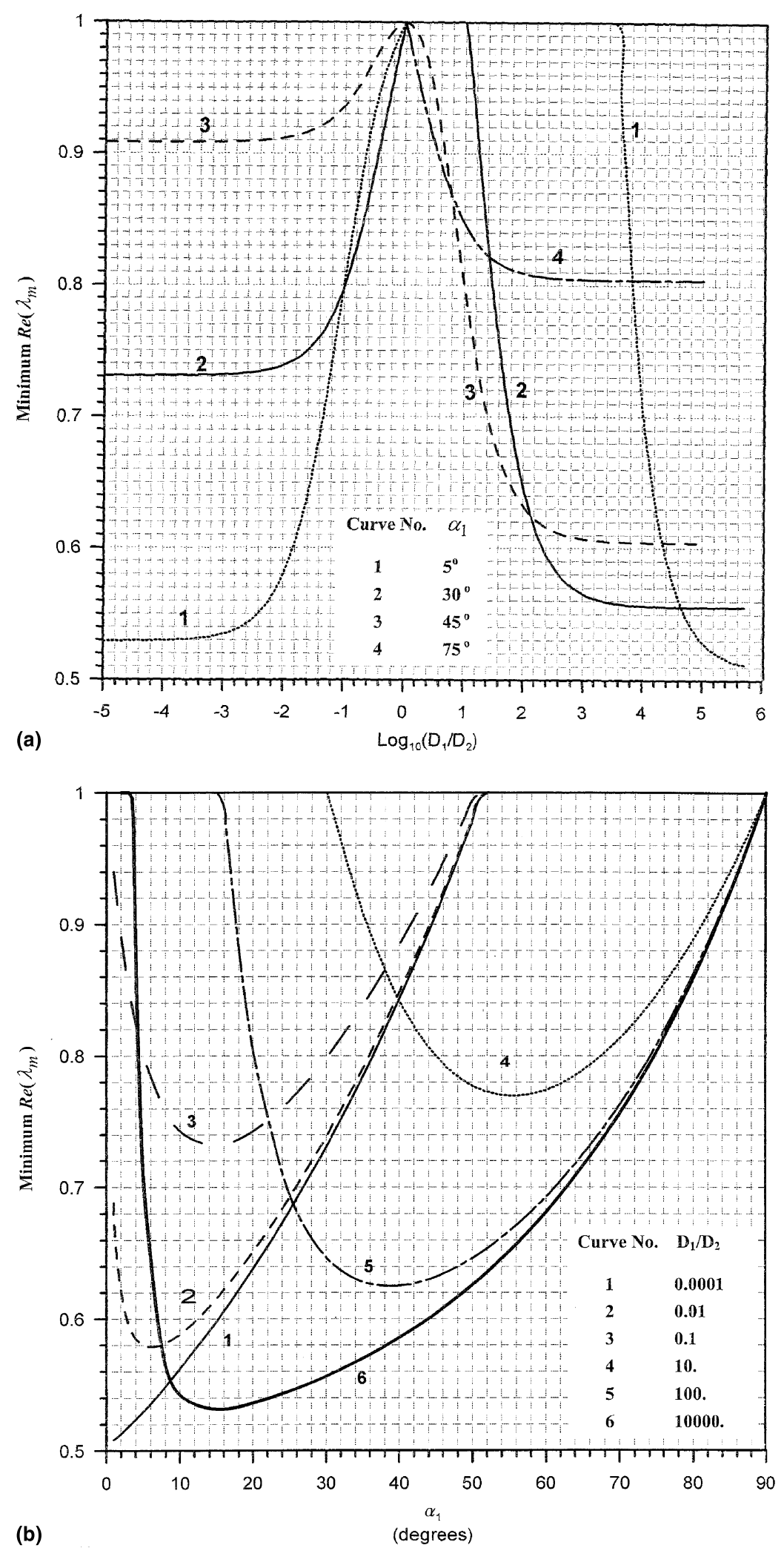

Fig. 6. (a) Variation of the minimum $\operatorname{Re}\left(\lambda_{m}\right)$ with $D_{1} / D_{2}$ for a wedge with $\alpha_{1}+\alpha_{2}=180^{\circ}$. (b) Variation of the minimum $\operatorname{Re}\left(\lambda_{m}\right)$ with $\alpha_{1}$ for a wedge with $\alpha_{1}+\alpha_{2}=180^{\circ}$. 


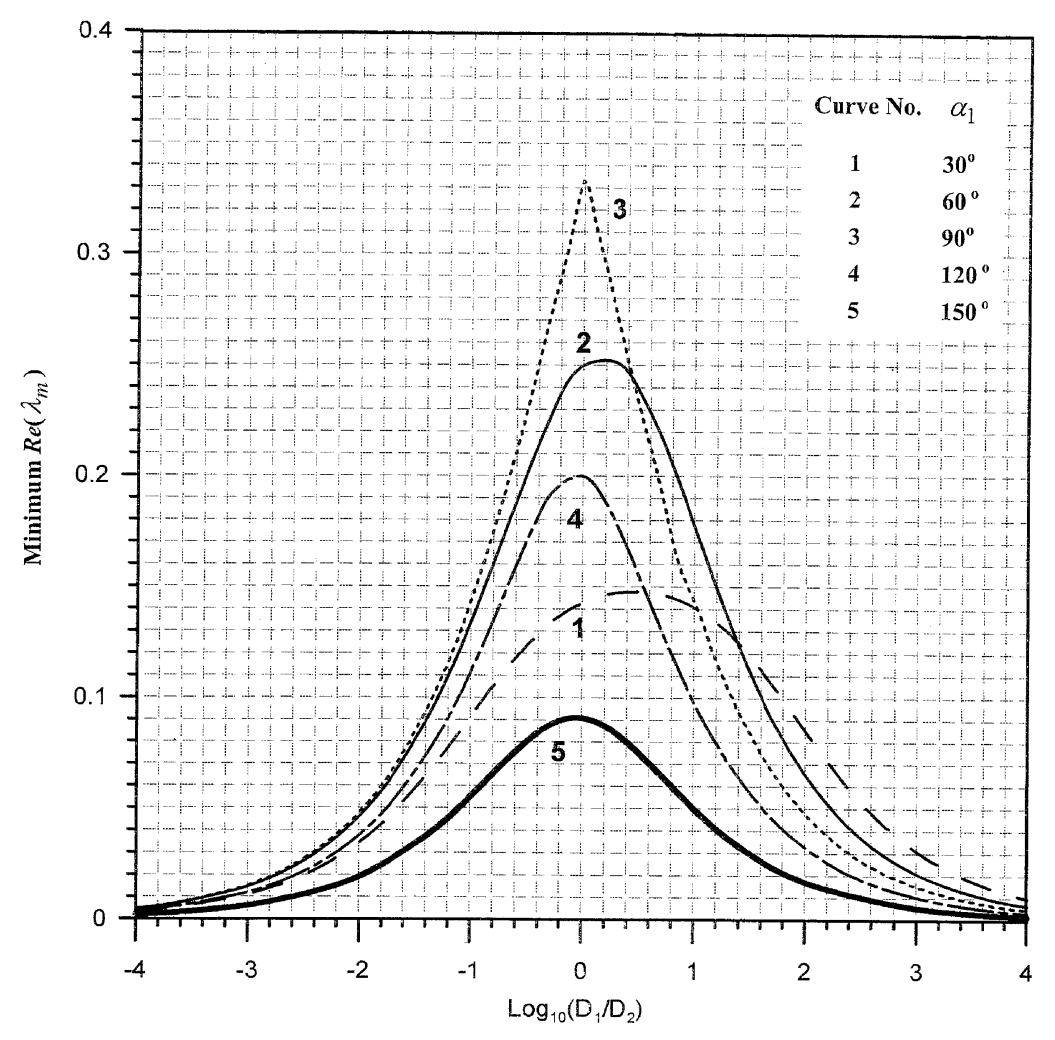

Fig. 7. Variation of the minimum $\operatorname{Re}\left(\lambda_{m}\right)$ with $D_{1} / D_{2}$ for a wedge with $\alpha_{2}=180^{\circ}$.

$\alpha_{2}=180^{\circ}$. These results reveal that the singularity always occurs. The weakest singularity for each $\alpha_{1}$ value occurs in the range of $D_{1} / D_{2}$ between 1 and 5. As $D_{1} / D_{2}$ moves further away from that range, the singularity becomes stronger.

\subsubsection{Characteristic equation for shear force singularity}

Substituting the solution given in Eqs. (12a)-(12c) into the boundary conditions and continuity conditions, one is able to find the following characteristic equation for a shear force singularity:

$G_{2} h_{2} \cos \bar{\lambda}_{m} \alpha_{2} \sin \bar{\lambda}_{m} \alpha_{1}+G_{1} h_{1} \cos \bar{\lambda}_{m} \alpha_{1} \sin \bar{\lambda}_{m} \alpha_{2}=0$.

As found in the case of bonded dissimilar isotropic plates, the solution $\bar{\lambda}_{m}$ of the characteristic equation is dependent on $G_{1} h_{1} / G_{2} h_{2}$ and $\alpha_{1}$ (or $\alpha_{2}$ ), but is independent of Poisson's ratios.

It should also be mentioned that as $G_{1} h_{1} / G_{2} h_{2}$ or $G_{2} h_{2} / G_{1} h_{1}$ approaches zero, Eq. (22) degenerates to the characteristic equations for a wedge with clamped and simply supported radial edges and for a wedge with free and simply supported radial edges, which are consistent with those given in [11]. Furthermore, when $G_{1} h_{1}=$ $G_{2} h_{2}$, Eq. (22) degenerates to the characteristic equation for a wedge with simply supported radial edges as given in [11], which will produce the same shear force singularity as that found in the exact solution for the vibra- tions of sector plates with simply supported radial edges $[11,20]$.

Fig. 8 shows the variation of the minimum $\operatorname{Re}\left(\lambda_{m}\right)$ with $G_{1} h_{1} / G_{2} h_{2}$ for $\alpha_{1}=5^{\circ}, 30^{\circ}, 45^{\circ}$, and $75^{\circ}$ with $\alpha_{1}+\alpha_{2}=180^{\circ}$, while Fig. 9 depicts the variation of the minimum $\operatorname{Re}\left(\lambda_{m}\right)$ with $G_{1} h_{1} / G_{2} h_{2}$ for $\alpha_{1}=$ $30^{\circ}, 60^{\circ}, 90^{\circ}, 120^{\circ}$, and $150^{\circ}$ with $\alpha_{2}=180^{\circ}$. The results shown in Fig. 8 indicate that there is no shear force singularity for $G_{1} h_{1} / G_{2} h_{2}$ exceeding (or equal to) one, while the singularity always occurs for $G_{1} h_{1} / G_{2} h_{2}$ less than one. Furthermore, the singularity becomes stronger as $G_{1} h_{1} / G_{2} h_{2}$ decreases. For very small $G_{1} h_{1} / G_{2} h_{2}$, the singularity becomes stronger as $\alpha_{1}$ decreases.

The results shown in Fig. 9 reveal that the singularity always occurs for all $G_{1} h_{1} / G_{2} h_{2}$. The singularity becomes weaker as $G_{1} h_{1} / G_{2} h_{2}$ becomes larger. As $G_{1} h_{1} / G_{2} h_{2}$ gets smaller and approaches zero, the minimum $\operatorname{Re}\left(\lambda_{m}\right)$ approaches 0.5 , which is the characteristic value for a clamped-simply supported isotropic and homogeneous wedge with an opening angle equal to $180^{\circ}$ [11]. When $G_{1} h_{1} / G_{2} h_{2}$ becomes larger and approaches infinity, the minimum $\operatorname{Re}\left(\lambda_{m}\right)$ approaches one for $\alpha_{1}$ less than (or equal to) $90^{\circ}$, while the values of the minimum $\operatorname{Re}\left(\lambda_{m}\right)$ for $\alpha_{1}=120^{\circ}$ and $150^{\circ}$, respectively, approach the characteristic values for clamped-simply supported isotropic and homogeneous wedges with corresponding corner angles. 


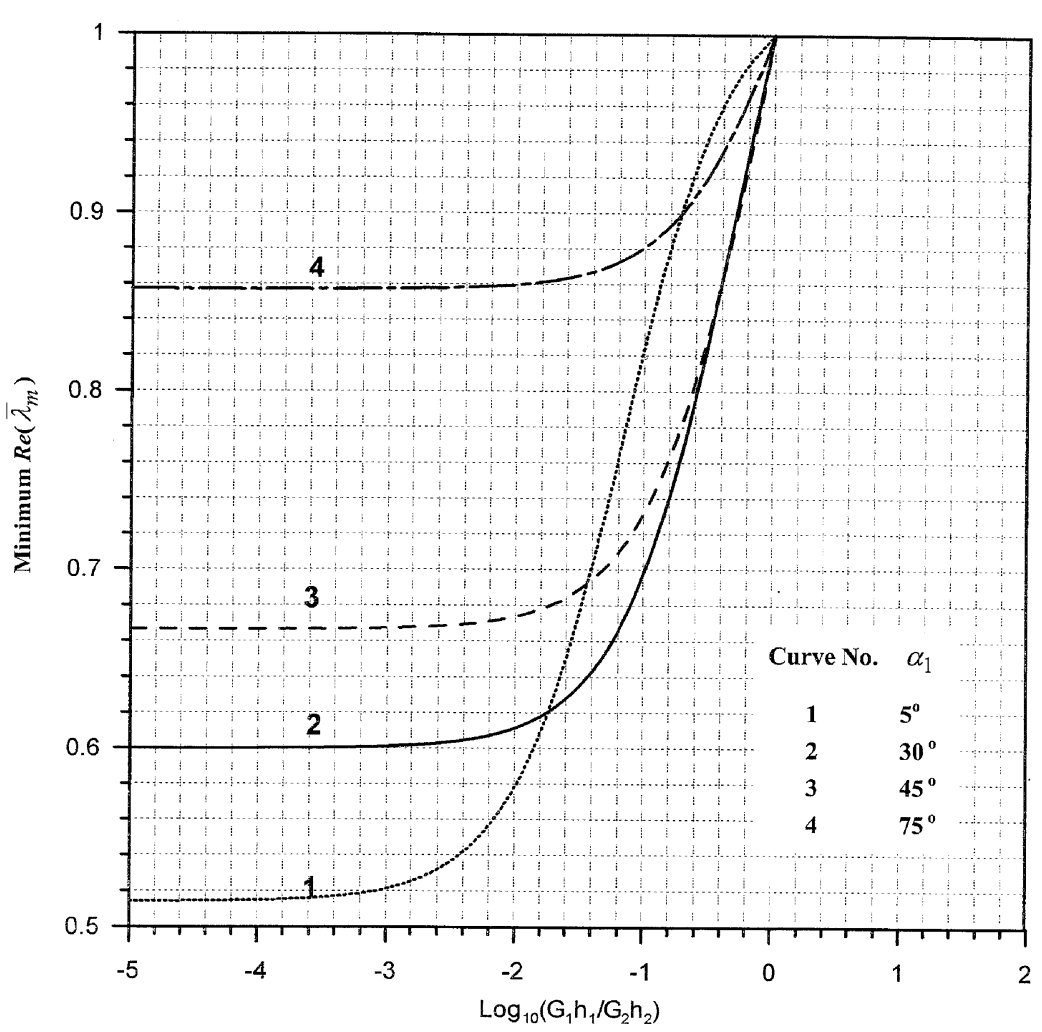

Fig. 8. Variation of the minimum $\operatorname{Re}\left(\bar{\lambda}_{m}\right)$ with $G_{1} h_{1} / G_{2} h_{2}$ for a wedge with $\alpha_{1}+\alpha_{2}=180^{\circ}$.

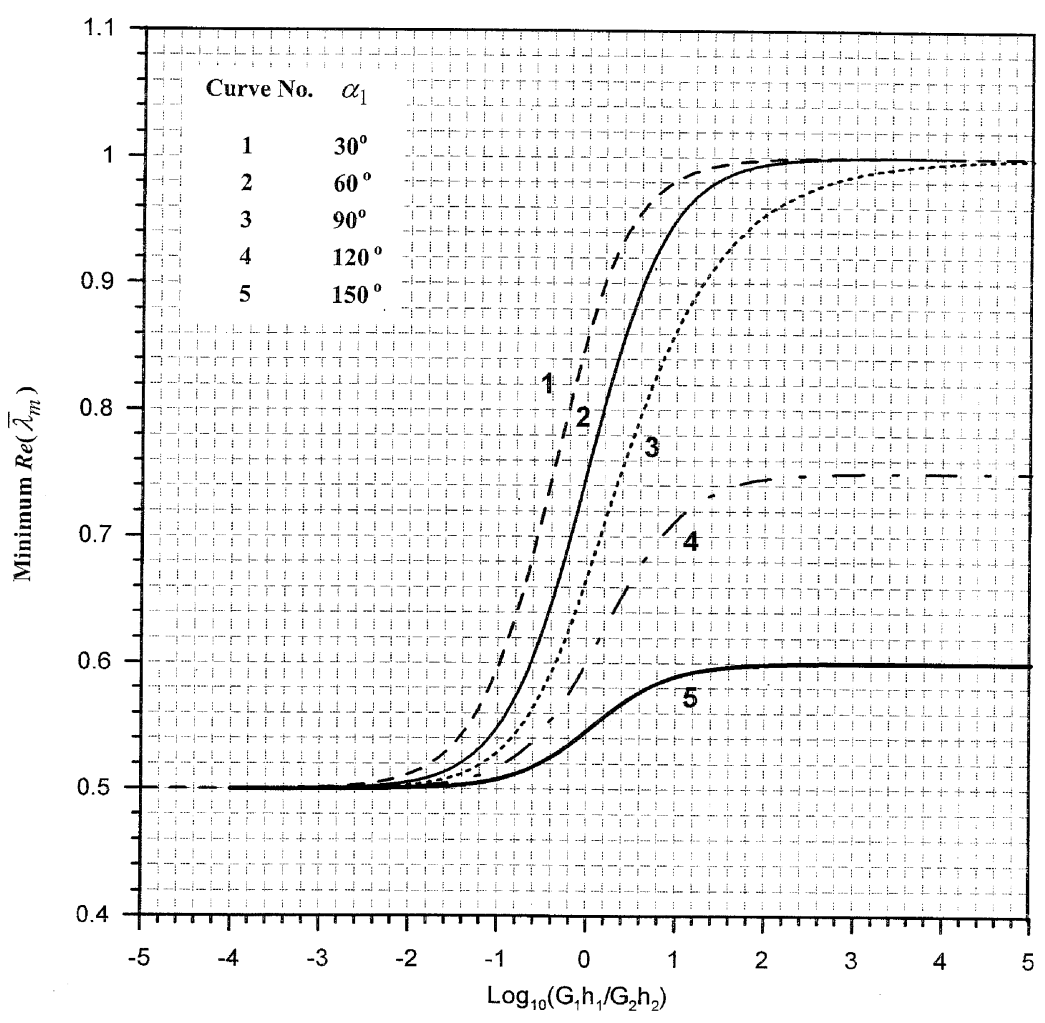

Fig. 9. Variation of the minimum $\operatorname{Re}\left(\bar{\lambda}_{m}\right)$ with $G_{1} h_{1} / G_{2} h_{2}$ for a wedge with $\alpha_{2}=180^{\circ}$. 


\section{Concluding remarks}

This paper has presented an eigenfunction expansion solution procedure for bi-material plates (wedges) based on the Mindlin plate theory. The stress resultant singularities of the William's type at the interface corner have been determined from the asymptotic solution satisfying radial boundary conditions and continuity conditions. The singularities of moments and shear forces have been independently investigated, starting from different solution forms.

The present solution procedure has been applied to investigate singularities at the interface corner of perfectly bonded dissimilar isotropic plates and singularities at the vertex of a bi-material wedge with simply supported radial edges. The first known characteristic equations for determining the singularity orders of moments and shear forces have been explicitly given. The order of moment singularity is dependent on the flexural rigidity radio $\left(D_{1} / D_{2}\right)$, Poisson's ratios $\left(v_{1}\right.$ and $\left.v_{2}\right)$, and corner angles for each material $\left(\alpha_{1}\right.$ and $\left.\alpha_{2}\right)$, while the order of shear force singularity depends on $G_{1} h_{1} / G_{2} h_{2}, \alpha_{1}$ and $\alpha_{2}$. However, it seems that the singularity order of moment is not significantly affected by Poisson's ratios. In certain special cases, such as when $D_{1} / D_{2}$ or $G_{1} h_{1} / G_{2} h_{2}$ approaches zero, these characteristic equations degenerate to those for an isotropic and homogeneous wedge with corresponding radial boundary conditions. The singularity order for the Mindlin plate theory is generally different from that for the classical plate theory, based on a comparison of the results for bonded dissimilar isotropic plates.

The present solution procedure can be easily extended to find the singularity orders of moments and shear forces for wedges with other radial boundary conditions or wedges composed of more than two materials. The asymptotic expression for displacement components that results in moment and shear force singularities can also be further developed. The obtained singularity orders for moments and shear forces, and the asymptotic displacement fields will be very important and useful for further numerical analysis (i.e., using the finite element approach or Ritz method) of complex problems involving multi-material corners.

\section{Acknowledgements}

This work reported herein was supported by the National Science Council, ROC through research grant no. NSC90-2211-E-009-035. This support is gratefully acknowledged. The appreciation is also extended to author's graduate students, Mr. K.H. He, Mr. M.C. Yang, and Mr. M.J. Chang, for preparing the figures shown in the paper.

\section{References}

[1] Leissa AW. Singularity considerations in membrane, plate and shell behaviors. Int J Solids Struct 2001;38:3341-53.

[2] McGee OG, Leissa AW, Huang CS. Vibrations of cantilevered skewed plates with corner stress singularities. Int $\mathbf{J}$ Numer Meth Eng 1992;35:409-24.

[3] Leissa AW, McGee OG, Huang CS. Vibrations of circular plates with V-notches or sharp radial cracks. J Sound Vib 1993;161:22739 .

[4] Huang CS, McGee OG, Leissa AW, Kim JW. Accurate vibration analysis of simply supported rhombic plates by considering stress singularities. J Vib Acoust 1995;117:245-51.

[5] William ML. Stress singularities resulting from various boundary conditions in angular corners of plates under bending. In: Proceedings of 1st US National Congress of Applied Mechanics, 1952. p. 325-9.

[6] William ML. Stress singularities resulting from various boundary conditions in angular corners of plates in extension. J Appl Mech 1952;19:526-8.

[7] William ML, Chapkis RL. Stress singularities for a sharp-notched polarly orthotropic plate. In: Proceedings of 3rd US National Congress of Applied Mechanics, 1958. p. 281-6.

[8] Ting TCT, Chou SC. Edge singularities in anisotropic composites. Int J Solids Struct 1981;17:1057-68.

[9] Ojikutu IO, Low RO, Scott RA. Stress singularities in laminated composite wedge. Int J Solids Struct 1984;20:777-90.

[10] Burton WS, Sinclair GB. On the singularities in Reissner's theory for the bending of elastic plates. J Appl Mech 1986;53:220-2.

[11] Huang CS. Stress singularities in angular corners in first-order shear deformation plate theory. Int. J. Mechanical Science 2002 [submitted].

[12] Hartranft RJ, Sih GC. The use of eigenfunction expansions in the general solution of three-dimensional crack problems. J Math Mech 1969;19:123-38.

[13] Hein VL, Erdogan F. Stress singularities in a two-material wedge. Int J Fract Mech 1971;7:317-30.

[14] Bogy DB, Wang KC. Stress singularities at interface corners in bonded dissimilar isotropic elastic materials. Int J Solids Struct 1971;7:993-1005.

[15] Rao AK. Stress concentrations and singularities at interface corners. Z Angew Math Mech 1971;51:395-406.

[16] Dempsey JP, Sinclair GB. On the stress singular behavior at the vertex of a bi-material wedge. J Elasticity 1981;11:317-27.

[17] Xie M, Chaudhuri RA. Three-dimensional stress singularity at a bimaterial interface crack front. Compos Struct 1998;40: $137-47$.

[18] Mindlin RD. Influence of rotatory inertia and shear on flexural motion of isotropic, elastic plates. J Appl Mech 1951;18:31-8.

[19] Huang CS. Singularities in plate vibration problems. Ph.D. Dissertation, Ohio State University, Columbus, OH, 1991.

[20] Huang CS, McGee OG, Leissa AW. Exact analytical solutions for free vibrations of thick sectorial plates with simply supported radial edges. Int J Solids Struct 1994;31:1609-31. 